9

\title{
Using an Informal Ontology in the Development of a Planning and Control System - the Case of the Virtual Enterprise
}

\author{
António Lucas Soares, Jorge Pinho de Sousa, Américo Lopes \\ Azevedo, João Augusto Bastos \\ INESC / Manufacturing Systems Engineering Unit, Rua José Falcão, \\ 110 - 4300 Porto, Portugal \\ email: \{asoares, jsousa, aazevedo, jbastos\}@inescn.pt
}

\begin{abstract}
This paper describes a preliminary experience in trying to improve the communication and to reach language agreements in the context of a large R\&D trans-national project, comprising partners with different academic and industrial cultures. This kind of projects has difficulties in that, besides cultural and language problems, the project teams undertake work largely by themselves, with reduced interaction with each other. The use of ontologies has a great potential in reducing those problems. Within this research work our goal is to extend the communication role of an ontology towards the mediation of the development actors "world views". In doing so, we intend to improve the engineering of an intrinsically complex software system, particularly in the requirements identification, system specification and system design phases, overcoming some of the referred difficulties. It is presented an extract of the Virtual Enterprise ontology currently under construction, focusing in some core definitions relevant for the purpose of developing a distributed planning and control software system. An example concerning the development of such a system is described in order to demonstrate the usefulness of the ontology based systems development.
\end{abstract}

\section{Keywords}

Information systems development, ontologies, planning and control, virtual enterprise

\section{INTRODUCTION}

This paper aims at reporting on some preliminary experience in trying to improve the communication and to reach language agreements in the context of a three year RTD trans-national project (E.20544, X-CITTIC, 1996/1999 - A Planning and Control System for Semiconductor Virtual Enterprises). This project aims at designing a model for planning and controlling an industrial Virtual Enterprise (VE) that should lead to the design of an architecture and to the development or selection of a set of software tools to be tested on two of the partners which 
are large complex organisations on the semiconductor industry.

The project involves three industrial companies on the semiconductor sector (GEC Plessey Semiconductors, TEMIC, ALCATEL-MIETEC), one software company (Nimble), and three academic institutions (Imperial College, INESC, Fraunhofer Gesellschaft IPA). The semiconductor industry clearly has features of the so-called Virtual Enterprise, in particular concerning distributed sites and different ownership situations of the plants. The manufacturing processes involve, in general, a set of sites (that may be located in different regions and countries), subcontracting and outsourcing, and create therefore quite complex logistic problems that have to be explicitly taken into account in planning processes. Moreover, due to the increasing demanding requests of customers, in a highly competitive environment, it becomes very important to have flexible planning systems and reliable control mechanisms. Quick response to the customers needs and to unpredictable changes in production conditions is also a need.

An Ontology for the VE is being developed as a first step towards constructing a platform for agreement on concepts and on the terminology to be used inside the project, as well as a way to structure the concepts to be used in the design. This is an attempt to acquire, represent and explore knowledge, by providing a core of basic concepts and language constructs, with precise purposes, i.e. to facilitate the project progress and to improve its efficiency and effectiveness.

\section{A PLANNING AND CONTROL SYSTEM FOR THE VIRTUAL ENTERPRISE}

Many manufacturing enterprises are becoming global businesses covering multiple manufacturing sites consisting of shop floors, subcontractors and suppliers. For that reason, planning and control activities are very complex, and have to take place both within the enterprise and across the whole supply network in order to achieve high levels of performance. However, this planning and control task is not covered well by state-of-the-art solutions. Moreover, the logistics associated to manufacturing products in several different plants and subcontracted companies become an important practical issue.

\subsection{Project Objectives}

The main deliverable of the current project will be a planning, scheduling and control system aiming at improving manufacturing performance in a virtual enterprise by integrating the heterogeneous manufacturing systems and by bridging the gap between the higher planning levels and the lower shop floor control levels. The project addresses the problem of planning and control of a distributed enterprise and aims at providing an integrated toolbox for distributed planning and scheduling that can be used in the operational control of manufacturing processes.

This toolbox will provide manufacturing solutions and services leading to shorter customer order lead times, improved delivery precision, reduced inventory and stocks, improved resource utilisation, quick response to customer enquiries, cost reduction in the logistic processes.

These targets will be realised by implementing: 
- means for improved customer due date calculation;

- a multi-site planning tool to co-ordinate local activities across the virtual enterprise network;

- an integrated toolset for local planning and control as well as the management of shop floor deviations and contingencies based on the selection of existing tools;

- the complementary development of missing links and an integration methodology;

- the consolidation of the distributed schedules through methodologies and tools for the negotiation between different modules;

- an integrating infrastructure for planning and scheduling, supporting co-operative software applications;

- novel interfacing capabilities with existing systems;

- a real-time information link bridging the gap between planning and shop floor execution processes.

Major advances will be required over state-of-the-art and will lead to new concepts and tools for distributed planning and scheduling. In addition, system openness, interfacing to other enterprise IT systems and hardware vendor independence will all be given the utmost priority in order to facilitate future flexibility to change.

\subsection{Project context}

The different partners have various cultures, with normal problems in "natural" language communication. Concerning the industrial partners, the different companies have organisations and languages that can be substantially different. Even if the general objectives and goals of the system under development were set in a precise manner, this was not the case of its scope. The very nature of the project, involving the idea of Virtual Enterprise, leads to difficulties in defining the entities covered by the concept and the roles to be played by those entities.

From the point of view of the project management there are obvious difficulties. The project teams work largely by themselves, with reduced interaction with each other. Periodical technical meetings are held with the purpose of aligning objectives and synchronising the various tasks. These co-ordination aspects are in practice an additional problem in terms of an efficient and smooth course of the project.

\section{ON THE CONCEPT OF VIRTUAL ENTERPRISE}

In the past, manufacturing was essentially organised on a local level. With the fast development of transportation and communication means, an expansion process took place across countries and continents, naturally leading to the creation of global markets and global manufacturing systems. Nowadays, more and more companies are being organised as manufacturing networks of different units, namely, plants, logistic centres and storage facilities. In order to produce cost effective products within the time scales requested by the customers, most of the companies are using subcontractors spread all over the world.

The concept of Virtual Enterprise was born inside this environment, but it is obviously ambiguous and hard to define. We should however try to set a precise definition, for operational and communication purposes. In the literature, we can find several approaches to these issues, 
but they are not completely satisfactory in solving the ambiguity and imprecision associated to the terms used in the domain. First of all, it is interesting to review some terms related to the VE. The APICS dictionary defines Virtual Corporation and Virtual Factory (APICS, 1995):

"Virtual Corporation - (...), the capabilities and systems of the firm are merged with those of the suppliers, resulting in a new type of corporation where the boundaries between the suppliers systems and those of the firm seem to disappear.(...).

Virtual Factory - (...). It is a transformation process that involves merging the capabilities and capacities of the firm with those of its suppliers.(...)."

Based on the GERAM (Generic Enterprise and Reference Architecture and Methodology) entities, Ferreira (1995) presents a framework that allows the consideration of the extended enterprise. This is achieved by labelling products, the manufacturing enterprise, the engineering design enterprise, and the strategic enterprise, as new entities of the reference architecture. In this context, the concept of extended enterprise encompasses not only the integration of processes from different manufacturing enterprises, but also their integration with the engineering enterprise and the strategic management enterprise. As one would expect, it may happen that the previously mentioned entities are in fact different enterprises, thus reinforcing the need for integration within the extended enterprise.

In Browne et al. (1995) the concept of extended enterprise is also explored. The extended enterprise is viewed as "an expression of the market driven requirements to embrace external resources in the enterprise without owing them". The emphasis is put in the integration of manufacturing and distribution planning and control systems. A similar concept is that of supply chain. For EIL (1995) the supply chain of a manufacturing enterprise is a world-wide network of suppliers, factories, warehouses, distribution centres through which raw materials are acquired, transformed and delivered to customers.

Another related interesting concept is that of network organisation in which separate firms, each retaining its own authority in major budgeting and pricing matters, function as integral parts of a greater organisation co-ordinated by a core firm. In this context, the different firms, spread along a value-added chain (e.g., suppliers, manufacturers, and distributors), tend to function in a way that stresses complementary, ongoing relationships, and reciprocity (Ching et al., 1993).

In the literature, much more related definitions and concepts can be found, and in the context of this project we came to the following first operational definition: "The Virtual Enterprise is a subset of units and processes within the supply chain which behave like a single company through strong co-operation towards mutual goals".

First, it is clear that we are talking of a physically distributed network of units (several sites) and possibly (likely) owned by different companies. Secondly, it should also be clear that we focus on the manufacturing aspects of the company, or on aspects that are strongly related to manufacturing. However these ideas are probably not crucial, as they do not capture the key features for planning / control purposes (which are the concerns of the project). As counter-examples, one can consider several distinct production units (supposed to be managed separately) standing on the same site, or the ability to largely control subcontractors not really owned. Therefore, as a first, non-formalised approach, we propose a slightly different view on the concept, based on the following ideas. We start by considering a set of units: these are separately managed entities (plants, subcontractors, transport companies, warehouses, "customers", ...) that have some kind of relationship to our business. As opposed to the concept of "node" (in a network) that has a "mathematical" connotation, the term "unit" better reflects the manufacturing terminology. For each of these units, we define the level-of- 
control (loc), in some rough, partially subjective way, say in 0-1 scale. This means that our core company has a loc of 1 , a given plant may have a loc of 0.7 , a subcontractor a loc of 0.3 , a "customer" might have a loc of 0.1 or close to 0 . Then we have, for the different units, some kind of "measure" of "ownership" towards the main (core) management entity (of course, this is not directly related to real ownership). This setting should allow us to define the VE in a kind of "fuzzy" way, not imposing a strict definition of the borders (type of "you are inside the VE, or you are out in the world...).

Nevertheless, for management purposes, it is important to set a "threshold", i.e. a minimum level of "ownership", below which we would say the unit is outside of the VE even if we can not forget it in our management processes (this should be the case of "normal" customers, but not the case of very closely related special customers, or some with whom partnerships have been established). In this context, a subcontractor could be of different types (related to his $l o c$ ), allowing different levels of information share and control.

It should be noted that the VE Ontology, currently under construction, does not yet formalise these ideas, but we feel they can be usefully incorporated and used in practice.

\section{THE NEED FOR MEDIATION}

In section 2 we saw how the specific case of a planning and control system development for a Virtual Enterprise run into difficulties given the profusion of concepts and terms and the confrontation of different perspectives. Our experience showed what others had already reported, in a different context, that terminological confusion breeds conceptual confusion and viceversa (Bradshaw et al.). This is mostly true in phases of conceptual brainstorming and perspectives confrontation which characterise the early phases of the system development process. One way to improve this process is to agree a priori with a conceptual and terminological core, setting the ground for the subsequent discussion. Such a core would assume a mediation role between the development actors. This led us to the use of ontologies in implementing the referred mediation scheme. An example of mediation in a broader techno-organisational context is reported in (Soares and Mendonça, 1996).

Ontologies are an active research area in the field of Artificial Intelligence supporting a modelling view of knowledge engineering as opposed to a transfer view (Guarino, 1995). In this perspective knowledge engineering is viewed not as the capture and storage of something extracted from an expert's mind but as the result of a constructive modelling process of an objective reality. Different definitions of ontology can be found in the literature. A concise definition that is becoming widely accepted is the one proposed by Gruber (1993): "an explicit specification of a conceptualisation". A good synthesis of what an ontology is appears in (Uschold and Gruninger, 1996), quoting a source in the SRKB mailing list: "Ontologies are agreements about shared conceptualisations. Shared conceptualisations include conceptual frameworks for modelling domain knowledge, content specific protocols for communication among inter-operating agents and agreements about the representation of particular domain theories. In the knowledge sharing context, ontologies are specified in the form of definitions of representational vocabulary" (Gruber, 1994).

\subsection{Purpose of the Ontology}

Ontologies have been applied so far in a range of works with several purposes. Knowledge sharing and reuse are the base line directly or indirectly referred in each of them. Sharing arises 
when a common conceptualisation of a given domain is essential to the undertaking and coordination of activities within that domain. Sharing implies some sort of communication between different people, people and implemented computational systems, different implemented computational systems (Uschold, 1995). Reuse can be viewed as the step forward towards a generalised sharing, through formalisation mechanisms. Our purpose in building an ontology for this project is to improve the communication between partners concerning the requirements identification, specification and design phases of the planning and control system. With this we expect to achieve a faster agreement on the system's conceptual model and a more consistent use of terms and concepts throughout the software development. Eventually, when a more mature state of this ontology is reached, we intend to codify it and make it available for reuse, in so contributing for the clarification of the Virtual Enterprise concept. As a side effect, we also expect to contribute to the research of a less explored role of an ontology: the one of communication medium between people.

\subsection{Issues in the Ontology Development}

In the construction of the Virtual Enterprise Ontology we followed whenever possible the methodology for developing ontologies outlined by Uschold and King (1995). This methodology includes the following steps: identify purpose, build the ontology (capture, code, integrate existing ontologies), evaluation and documentation. According to our goals, we concentrated in the purpose identification and building steps, particularly the capture phase. The later is probably a crucial step in the process, and consists in identifying the key concepts and relationships in the domain, producing precise text definitions, identifying terms, and reaching an agreement on these issues. Another important aspect in the capture phase is the inclusion/integration of other ontologies. In our case, the Enterprise Ontology (Uschold et al., 1996) was particularly helpful and was thoroughly used in the VE Ontology construction. The Plan Ontology (Tate, 1995), although in a draft state, was also used.

\section{AN EXTRACT OF THE VIRTUAL ENTERPRISE ONTOLOGY}

We present now an extract of the VE Ontology, focusing in some core definitions relevant for the purpose of developing a planning and control system. It should be noted that this ontology is still under construction, and dynamically evolving. At this stage, a certain degree of nonformalism is kept.

As a first step towards defining this ontology, we have started by an attempt to list all possible entities involved in what might be the VE. As a basis, we have taken concepts from a related, more precise idea, that of a Supply Chain. Then we have tried to match those concepts and terms with those in already existing more general ontologies, such as the Enterprise Ontology (EO). The VE Ontology is committed to the Meta-Ontology defined in the EO.

It should be noted these are still preliminary steps, intended to illustrate the general approach followed in this work. We adopted the following notation: words in upper case are terms defined in the VE Ontology; words in upper case and italics were included from other ontologies (their formal definition is not presented, as their usual interpretation is enough for the purposes of this explanation). 


\section{Networked/Extendend Organisations}

VIRTUAL ENTERPRISE: is a set of inter-related VE UNITS that are totally or partially committed to some common PURPOSE.

Notes:

1. The relation between VE UNITS can be one of OWNERSHIP or a looser one of belonging to a common supplier chain.

VE UNIT: An ORGANISATIONAL UNIT belonging to the set that composes a VIRTUAL ENTERPRISE. A VE UNIT may be a manufacturing/processing plant, a subcontractor, a transport provider, a supplier or a warehouse.

Notes:

1. The term $O U$ is here used in its broader sense i.e., as an UNIT providing the a complete set of products or services (e.g. a plant, a manufacturing unit, a warehouse, etc.)

Plans management

PLAN: an ACTIVITY SPECIFICATION with an INTENDED PURPOSE.

Notes:

1. See notes under ACTIVITY SPECIFICATION.

PLANNING: an ACTIVITY whose INTENDED PURPOSE is to produce a PLAN.

PRODUCTION PLAN: is a PLAN whose INTENDED PURPOSE is to specify when and where PRODUCTION ACTIVITIES will be performed. A PRODUCTION PLAN produces an assignment of ACTIVITIES to VE UNITS and TIME INTERVALS.

ROUGH PRODUCTION PLAN: is a PRODUCTION PLAN based on aggregate information and setting TIME INTERVALS for production in the VE UNITS considered to be feasible candidates.*

FINE PRODUCTION PLAN: is a PRODUCTION PLAN based on more detailed information, and setting CALENDAR DATE's for production in specific VE UNITS.*

GLOBAL PLANNING: an ACTIVITY whose INTENDED PURPOSE is to produce PRODUCTION PLAN's

LOCAL PLANNING: an ACTIVITY whose INTENDED PURPOSE is to produce PRODUCTION PLAN's for a single VE UNIT.

CAPACITY: is an ATTRIBUTE of a RESOURCE representing what is available for allocation to ACTIVITIES over time. The CAPACITY of a RESOURCE constrains the number of ACTIVITIES that it can simultaneously support.

RESOURCE: is an ENTITY with some amount of CAPACITY that is allocable to ACTIVITIES over time

Orders management

ORDER: it is a CUSTOMER request for PRODUCT, issued to a SUPPLIER.. An ORDER contains one or more ORDER ITEMS.

Notes:

1. An ORDER contains general information such as the CUSTOMER identifier, number of items, shipping address, etc.

ORDER ITEM: an item within an ORDER. This includes reference to the PRODUCT SPECIFICATION, the quantity of PRODUCT to produce, and the CUSTOMER's requested DELIVERY DATE. 
CUSTOMER ORDER: is an ORDER in which the CUSTOMER is an EXTERNAL CUSTOMER.

Notes:

1. A CUSTOMER ORDER can be in one of the following states: under negotiation, confirmed, planned, being executed, completed.

PRODUCTION ORDER: is an ORDER in which the CUSTOMER is an INTERNAL CUSTOMER.

Notes:

1. A PRODUCTION ORDER can be in one of the following states: under planning, planned, being executed, on schedule, late, stopped, cancelled and completed.

PRODUCT REQUEST: is a statement defining a CUSTOMER's needs, in terms of PRODUCT types, quantities and DUE DATES.

CUSTOMER: the requirer of a PRODUCT. He expresses is requirements in terms of ORDERS. A CUSTOMER can be an INTERNAL CUSTOMER or an EXTERNAL CUSTOMER depending on its commitment to the VIRTUAL ENTERPRISE.

SUPPLIER: a provider of goods or services.

PRODUCT: is the result of a manufacturing ACTIVITIES chain. A manufacturing enterprise PURPOSE is to make PRODUCTS.

ENQUIRY: is an ACTIVITY that an EXTERNAL CUSTOMER EXECUTES with the PURPOSE of obtaining information about PRODUCTS, prices and DELIVERY DATES. This process eventually leads to placing a COSTUMER ORDER.

QUOTATION: is a statement of price, description of PRODUCTS, DUE DATES, and other terms of sale, offered by a supplier to a prospective CUSTOMER.

DUE DATE: is the CALENDAR DATE when PRODUCTS are available for delivery.

DELIVERY DATE: is the CALENDAR DATE when PRODUCTS are delivered to the CUSTOMER.

ORDER PROMISING: an ACTIVITY whose PURPOSE is to produce a QUOTATION in response to an EXTERNAL CUSTOMER REQUEST.

AVAILABLE-TO-PROMISE: is a quantity of PRODUCT available to allocate in a given TIME POINT to satisfy a CUSTOMER REQUEST. This quantity is calculated on the basis of the company's inventory and actual and planned production.

\section{THE VE ONTOLOGY AS A MEDIATOR IN THE SYSTEM DEVELOPMENT LIFE-CYCLE}

As referred above, one less explored role of an ontology is the one of communication medium between different people working together for a given purpose. This is the case of a (software) system development process where teams composed by end users and system developers have to collaborate throughout the development life cycle. In this research work our goal is to extend the communication role towards the mediation of the development actors "world views". In doing so, we intend to improve the engineering of an intrinsically complex software system, particularly in the requirements identification, system specification and system design phases, overcoming some of the difficulties described before. 


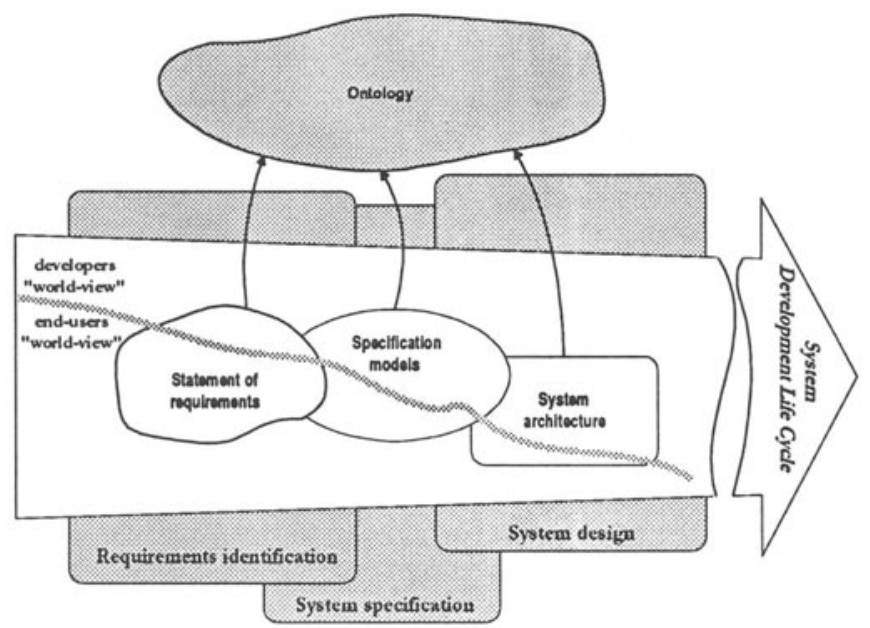

Figure 1 - Use of the VE Ontology in the Planning and Control system development

Figure 1 shows the general approach to the use of ontologies in the system development process. During the development life cycle end-users and developers world views constraint the purpose, scope and goals of the development object, as well as the discourse to describe and reason about the domain/system. Though the purpose, scope and goals of the system must be minimally agreed early in the project, conceptual differences and language misunderstandings and redundancies are important obstacles in a smooth evolving of the process. The degree of influence that each world view have in the development life cycle phases is qualitatively represented in the figure by the two areas separated by the thick grey line. End-users world-view has more influence in the requirements identification and specification phases whilst developers world view is more influential in the system design phase. System development phases and milestones are shown overlapping each other to symbolise both the fuzziness of the borders between phases and a desirable system development approach based on evolutionary prototyping. The role of the ontology as mediator is materialised in the concepts and terminology used in the system development milestones - statement of requirements, specification models and system architecture, which are expressed according to the terminology and structure defined by that ontology.

\subsection{An Example of Ontology Based Systems Development}

The example we are about to present is intended to demonstrate the usefulness of the ontology based systems development approach in improving the analysis, specification and design phases of the project. It is focused on a set of activities that can be designated by Global Planning and Control. We begin by transcribing the statement of requirements (at a very broad level) of for this set of activities using the terminology defined in the VE Ontology:

“...Global Planning and Control must include a set of activities designated by order promise that should respond to a CUSTOMER ENQUIRY by commitment to a DUE DATE, PRODUCT QUANTITY, PRODUCT QUALITY and PRODUCT PRICE i.e., a CUSTOMER QUOTATION. These promises are used to build up a rough PRODUCTION PLAN. A ROUGH PRODUCTION PLAN is a PLAN containing suggested TIME 
INTERVALS for production in the UNITS which are considered to be feasible candidates at an aggregate level. The ROUGH PRODUCTION PLAN is revised every time a CUSTOMER ENQUIRY is received or when an exception occurs requiring to repair the ROUGH PRODUCTION PLAN (see details on exceptions below).

The ROUGH PRODUCTION PLAN is used by a global PLANNING set of activities in the generation of a FINE PRODUCTION PLAN for each UNIT in the VIRTUAL ENTERPRISE. A FINE PRODUCTION PLAN is a PRODUCTION PLAN with a high degree of feasibility with respect to throughput, slack, conformance to the original PLAN and cost. The PURPOSE of the global PLANNING set of activities is to generate FINE PRODUCTION PLANS.

In order to adjust the ROUGH PRODUCTION PLANS and CUSTOMER QUOTATIONS in face of unexpected constraints a set of reactive ACTIVITIES are needed. This set of activities is designated by exceptions handling. Examples of exceptions may be: unrealised forecasts and unexpected UNIT CAPACITY change ..."

The statement of requirements is the starting point for a semi-formal specification of the planning and control system. To illustrate this, the well known OMT (Object Modelling Technology) methodology (Rumbaugh et al., 1991) was followed and its functional model used to translate the statement of requirements into the systems specification functional part. An extract of the model is shown in Figure 2. The use of the VE Ontology assures a conceptual and terminological coherence and consistence of concepts and terminology between the two milestones.

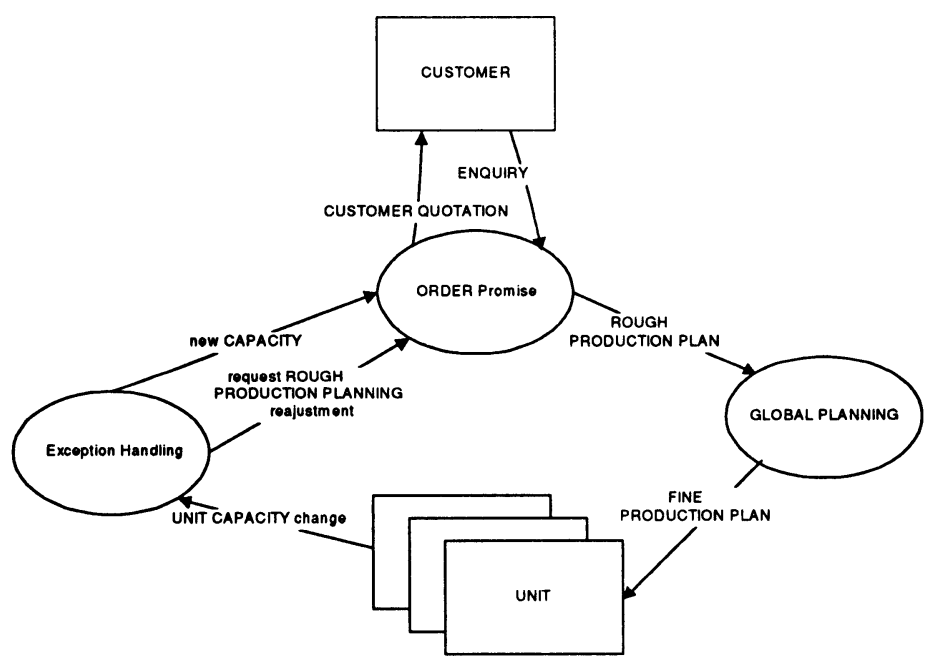

Figure 2 - Extract of the Global Planning and Control functional specification model (OMT)

\section{CONCLUSIONS AND FURTHER DEVELOPMENTS}

The preliminary experience reported in this paper shows that the use of an informal specifically created ontology has a great potential for mediating the development of large, complex software systems, by providing a platform for agreement on the language to be used inside the project, and also as a way to structure the concepts to be used in the design.

This approach was applied to a large R\&D trans-national project, involving partners with 
different academic and industrial cultures, aiming at designing a planning, scheduling and control system for the Virtual Enterprise (VE). Significant improvements are being obtained by using this approach, particularly in the requirements identification, system specification and system design phases.

As a first step towards constructing this ontology, the concept of Virtual Enterprise was fully discussed, leading to a clarification of the entities and terms to be used in the project.

The development of an ontology is a complex and time consuming task, that is dynamically evolving over time. The work done is far from having reached a stable and consolidated outcome. Further work is needed throughout the project, as well as the continuous improvement of the approach described in this paper.

\section{REFERENCES}

APICS, 1995, APICS Dictionary, $8^{\text {th }}$ Edition. American Production and Inventory Control Society, Inc.

Bradshaw, J.M., Boose, J.H., Shema, D.B., 1992, Steps Toward Sharable Ontologies for Design Rationale. AAAI-92 Design Rationale Capture and Use Workshop, San Jose, CA, July.

Browne, J., Sackett, P., Wortmann, H., 1994, Industry Requirements and Associated Research Issues in the Extended Enterprise. European Workshop on Integrated Manufacturing Systems Engineering, IMSE'94, 12-14, Grenoble.

Ching, C., Holsapple, C.W., Whinston, A.B., 1993, Modeling Network Organizations: A Basis for Exploring Computer Support Coordination Possibilities. Journal of Organizational Computing, 3(3), 279-300.

EIL (Enterprise Integration Laboratory), 1995, The Integrated Supply Chain Management Project. http://www.ie.toronto.ca/EIL/eil.html.

Ferreira, J.P., Mendonça, J.M, 1995, Modelling and Simulation of Manufacturing and Information Aspects of the Extended Enterprise. Proc. of the Int. Conf. on Improving Manufacturing Performance in a Distributed Enterprise: Advanced Systems and Tools, Edinburgh, U.K.

Gruber, T.R., 1993, A Translation Approach to Portable Ontology Specifications. Knowledge Acquisition, 2:199-220.

Guarino, Nicola, Formal Ontology, Conceptual Analysis and Knowledge Representation. To appear in International Journal of Human and Computer Studies, edited by N. Guarino and R. Poli.

Rumbaugh et al., 1991, Object-Oriented Modeling and Design, Prentice Hall.

Tate, A., 1995, Towards a Plan Ontology, Technical Report AIAI - The University of Edinburgh.

Uschold, M., King, M., 1995, Towards a Methodology for Building Ontologies. Technical Report AIAI-TR-183, The University of Edinburgh.

Uschold, M., King, M., Moralee, S., Zorgios, Y. The Enterprise Ontology. Technical Report AIAI-TR-195, The University of Edinburgh, 1996. [http://www.aiai .ed.ac.uk / entprise/enterprise/]

Uschold, M., Gruninger M., 1996b, Ontologies: Principles, Methods and Applications. Knowledge Engineering Review, 11 (2). 
Soares, A.L., Mendonça, J.M., 1996, Multiple-Perspective Knowledge Representation in Techno-Organisational Development Processes, in Koubek and Karwowski (Eds.), Manufacturing Agility and Hybrid Automation-I, IEA Press, Louisville, Kentucky, USA.

\section{BIOGRAPHY}

Antonio Lucas Soares is an Electrical Engineer teaching at the Faculty of Engineering of the University of Porto and doing research work at INESC-UESP. He holds a MSc. in Industrial Automation and is currently undertaking its $\mathrm{PhD}$ work in the area of collaboration models for techno-organisational development. The main research interests are the interdisciplinary collaboration in the development of integrated manufacturing systems and socio-technical systems.

Jorge Pinho de Sousa is an Assistant Professor at the Faculty of Engineering of the University of Porto and researcher at INESC-UESP. He holds a Ph.D. in Operations Research (Université Catholique de Louvain, Belgium), 1989. His main interests are on Operations Research, Combinatorial Optimization, Project Management, Industrial Organization, and in Production and Operations Management.

Américo Lopes de Azevedo obtained the degree of Electrical and Computers Engineer at the Faculty of Engineering of the University of Porto and is doing research work at INESC-UESP. He has been teaching in the Electronics and Computers Department where is assistant since 1988. Currently, he is preparing his $\mathrm{PhD}$ with a work in the area of manufacturing systems. His main interests lie in the Integrated Manufacturing area.

João Bastos obtained the degree of Mechanical Engineer at the Faculty of Engineering of the University of Porto and is doing research work at INESC-UESP. He holds a MSc. in Industrial Informatics and is currently undertaking his $\mathrm{PhD}$ work in the area of intelligent manufacturing systems. 